\title{
Can High Preoperative Neutrophil-lymphocyte Ratio Predict the Recurrence and Progression Risk of Non- muscle-invasive Bladder Tumors?
}

\author{
(1) Fuat Kızılay MD, ๑ Adnan Şimşir MD \\ Ege University Faculty of Medicine, Department of Urology, Izmir, Turkey
}

\begin{abstract}
Objective: Neutrophil-lymphocyte ratio (NLR) is a well-known, cost-effective biomarker of inflammatory conditions, and its protumor effect has been shown in different types of cancers. In this study, we aimed to evaluate the relationship between blood parameters, especially NLR, with the risk of progression and recurrence in non-muscle-invasive bladder tumors (NMIBT).

Materials and Methods: Seventy-six patients were included in the study. Patients were divided into low, moderate and high-risk groups according to the risk of progression and recurrence. The preoperative blood parameters of the patients were recorded from the patient files and the NLR of each patient was calculated. These parameters were compared in terms of progression and recurrence risk groups. P values less than 0.05 were accepted statistically significant.

Results: Neutrophil-lymphocyte ratio was significantly higher in the high-risk group in both the progression and recurrence risk groups than in the low and moderate risk groups $(\mathrm{p}<0.001)$. In addition, according to the post hoc results, the NLR values in the high-moderate and moderate-low risk groups showed significant differences (high-moderate and moderate-low values in terms of risk of recurrence were 4.66 vs 3.67 and 3.67 vs 2.88 , respectively, $\mathrm{p}<0.001$; high-moderate and moderate-low values in terms of risk of progression were 4.72 vs 3.68 and 3.68 vs 2.92 , respectively, $\mathrm{p}<0.001)$.

Conclusion: In our study, we found that groups with high risk of recurrence and progression had higher NLR values in patients with NMIBT. NLR, which is cheap, rapid and routinely applied in preoperative evaluation, is a promising biomarker in the prognostic classification of bladder tumors. Well-designed, large-scale prospective studies with long-term follow-up are needed to determine the role of NLR in this issue.

Keywords: Neutrophil-lymphocyte ratio, non-muscle-invasive bladder tumor, progression, recurrence, prognosis
\end{abstract}

\section{Introduction}

Bladder tumor (BT) is the most common malignancy of urinary tract with the highest incidence, and it is the seventh most common malignancy in men and eleventh most common malignancy in both genders (1). Approximately $75 \%$ of BT is limited to mucosa (Ta), carcinoma in-situ (CIS) or submucosa (T1) at the time of diagnosis, and this rate may be higher in patients younger than 40 years (2). Treatment in non-muscleinvasive bladder tumors (NMIBT) is planned according to the prognostic characteristics of the disease. According to the scoring system and risk tables developed by the genito-urinary cancer group of European Organization for Research and Treatment of Cancer (EORTC), the number of tumors, tumor size, previous recurrence rate, $\mathrm{T}$ stage, presence of carcinoma in-situ (CIS) and tumor grade are the most important factors predicting the possibility of tumor progression and recurrence (3). Studies were carried out to determine the factors that predicted the prognosis of NMIBT and a new scoring system was established by the Spanish Urological Club for Oncological Treatment after the analysis of 1062 patients. In addition to EORTC's scoring system, the age and gender of the patient were also included in the evaluation (4). Then, in their analysis including 1812 patients who received maintenance BCG treatment, EORTC stated that previous recurrence rate and number of tumors were the most important prognostic factors for disease recurrence and that stage and grade were the most important prognostic factors for disease progression, nomograms were designed according to new risk groups (5). 
Since NMIBT is a heterogeneous disease group with different recurrence, progression and disease-related mortality rates, the planned treatment and follow-up protocol may vary according to the risk classification and preferences of the patients. Therefore, it is very important to determine the variables that can predict the risk of recurrence and progression of patients and to plan the appropriate treatment for each patient group (3).

The systemic inflammatory condition triggered by cancer cells causes neutrophilia and lymphocytopenia, resulting in a tumorigenic inflammatory environment. Neutrophillymphocyte ratio (NLR) is a well-known and cost-effective marker of inflammatory conditions. In addition to many inflammatory conditions, high NLR has been shown to cause a worse prognosis in many different cancers such as colon, pancreas, stomach and lungs $(6,7,8,9)$. High preoperative NLR has been shown to cause poor prognosis and pathological stage progression in bladder tumors (10). However, in most of these studies, the tumors investigated in relation to high NLR are muscle-invasive tumors and it has been shown that the increased NLR in these studies is related to the presence of muscle invasion, extravesical disease and poor cancer-specific and overall survival $(10,11,12)$.

The aim of this study was to investigate whether high NLR is a determinant factor for progression and recurrence in patients with a diagnosis of NMIBT with transurethral resection of BT (TURB) and histopathological confirmation of non-muscleinvasion $(\mathrm{Ta}, \mathrm{T} 1)$.

\section{Materials and Methods}

\section{Patient Selection and Study Design}

The data of 122 patients who were operated on in our clinic between January 2016 and June 2018 with a diagnosis of BT were retrospectively analyzed. A total of 46 patients were excluded from the study, including 27 patients with confirmed histopathological diagnosis of MIBT, six patients with an active infection at the time of diagnosis, four patients with a hematological disease and nine patients with systemic inflammatory disease such as Systemic Lupus Erythematosus,

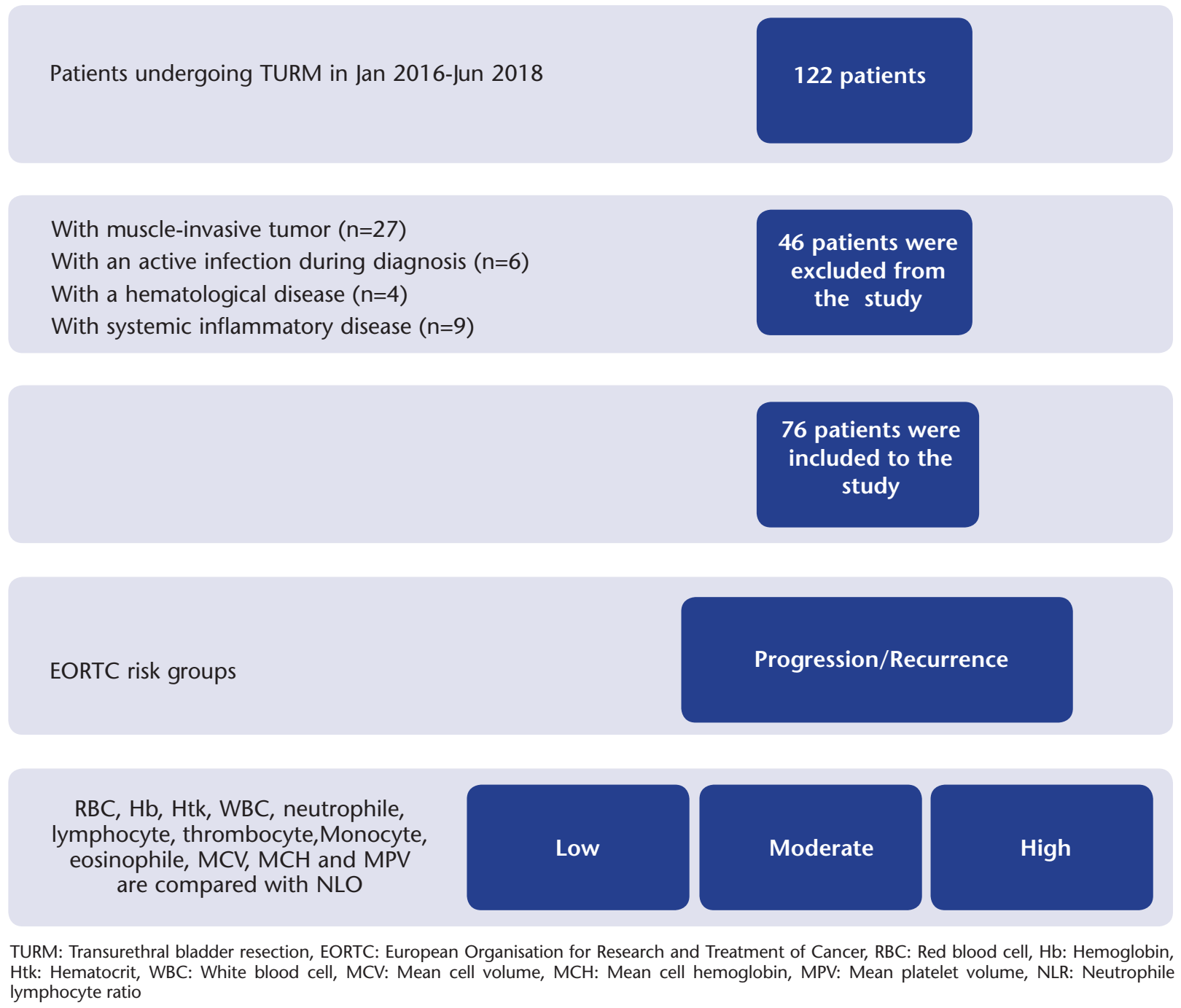

Figure 1. The flow chart of the study 
Behçet or Sjögren that may affect NLR. The remaining 76 patients were included in the study. The red blood cell (RBC), hemoglobin $(\mathrm{Hb})$, hematocrit ( $\mathrm{Htc})$, white blood cell (WBC), neutrophil, lymphocyte, platelet, monocyte, eosinophil, mean cell volume (MCV), mean cell hemoglobin $(\mathrm{MCH})$ and mean platelet volume (MPV) before TURB were recorded from patient files and NLR of each patient was calculated. Based on the histopathology of the patients after the first TURB operation, further treatments were performed as specified in the European Association of Urology (EAU) guidelines (intravesical chemotherapy or immunotherapy for NMIBT, radical cystectomy for MIBT) $(13,14)$.

Patients were divided into low, moderate and high-risk groups according to the progression and recurrence risks after a mean follow-up period of 18.4 months. The patients' risk score for recurrence and progression was made according to the EORTC classification system based on the number of tumors, size, previous recurrence rate, $\mathrm{T}$ stage, concomitant $\mathrm{CIS}$ and tumor grade. Progression risk groups were as follows: low-risk group: a score of 0, moderate-risk group: score between 2-6 and highrisk group: score $>7$. Recurrence risk groups were as follows: low-risk group: a score of 0 , moderate risk group: score between 1-9 and high risk group: score $>10(3,13)$.

The primary outcome of the study was the evaluation of the relationship between NLR and the risk of progression and recurrence, and the secondary outcome was the evaluation of the relationship between blood parameters and the risk of progression and recurrence. Before the operation, written informed consent was obtained from all patients in order to be able to use their data in scientific studies without revealing their private information. The flow chart of the study is shown in Figure 1.

\section{Statistical Analysis}

Descriptive statistics were given as mean \pm standard deviation (SD). The Shapiro-Wilk test was used to check the normality of

\begin{tabular}{|c|c|c|c|c|c|c|}
\hline \multicolumn{3}{|c|}{ Recurrence risk $(n=76)$} & \multicolumn{3}{|c|}{ Progression risk $(n=76)$} & \multirow{2}{*}{$\begin{array}{l}\mathbf{p} \\
0.249\end{array}$} \\
\hline \begin{tabular}{|l} 
Low \\
12 \\
22 \\
\end{tabular} & $\begin{array}{l}\text { Moderate } \\
10 \\
16\end{array}$ & $\begin{array}{l}\text { High } \\
6 \\
10 \\
\end{array}$ & \begin{tabular}{|l} 
Low \\
15 \\
26 \\
\end{tabular} & $\begin{array}{l}\text { Moderate } \\
8 \\
14\end{array}$ & $\begin{array}{l}\text { High } \\
5 \\
8 \\
\end{array}$ & \\
\hline $52.8 \pm 3.4$ & $56.6 \pm 4.2$ & $61.4 \pm 5.8$ & $53.9 \pm 4.6$ & $58.8 \pm 5.4$ & $63.2 \pm 5.9$ & 0.026 \\
\hline $21.4 \pm 3.8$ & $23.8 \pm 2.9$ & $22.6 \pm 2.1$ & $23.3 \pm 3.1$ & $24.9 \pm 2.0$ & $23.8 \pm 4.2$ & 0.488 \\
\hline $0.8 \pm 0.3$ & $1.6 \pm 1.1$ & $2.3 \pm 1.4$ & $1.0 \pm 0.4$ & $2.1 \pm 0.8$ & $3.2 \pm 0.9$ & 0.032 \\
\hline
\end{tabular}

Table 2. Comparison of blood parameters of patients according to risk tables developed by European Organization for Research and Treatment of Cancer (EORTC) genito-urinary cancer group

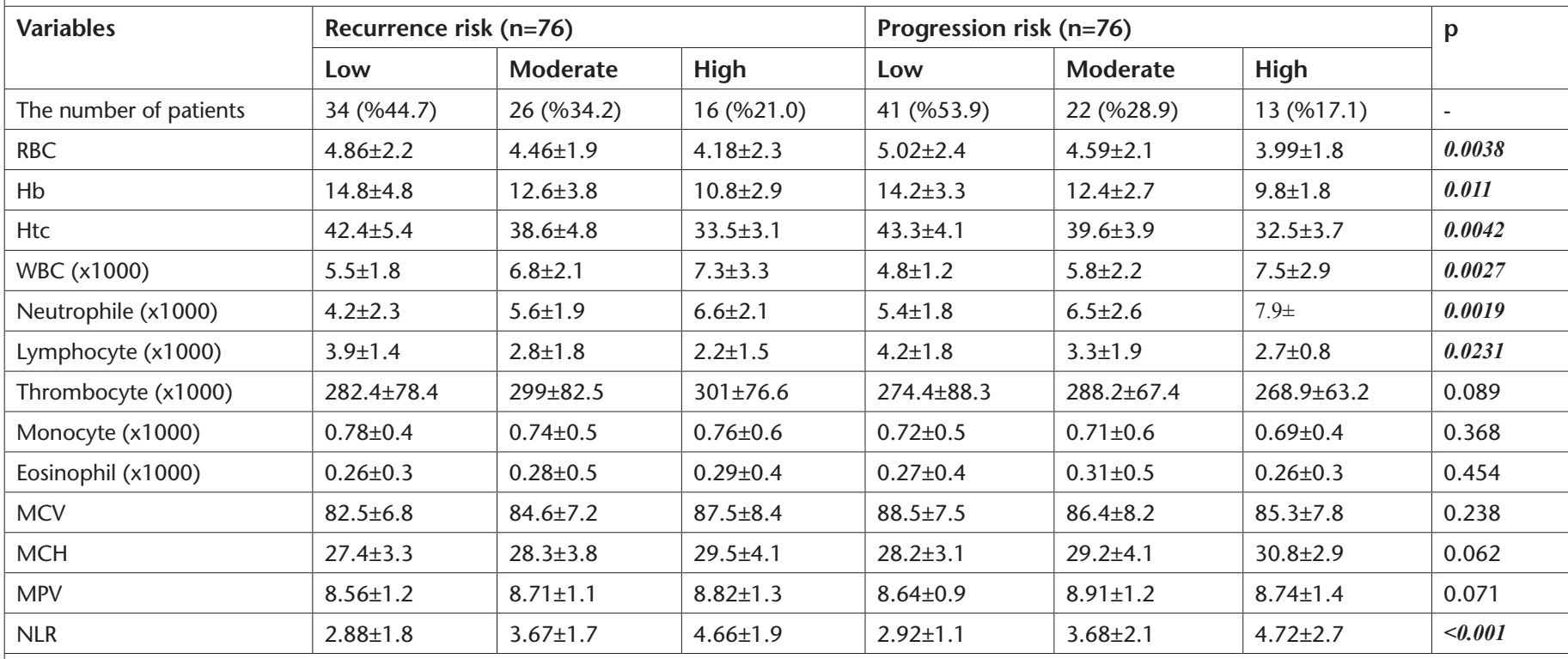

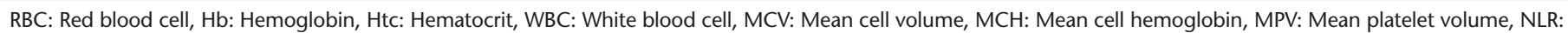
Neutrophil-lymphocyte ratio; Values are given as mean \pm SD or as number (\%)

Statistically significant $p$ values were given in bold and italics. 
the distribution. The patient characteristics of the three groups were compared using Pearson's chi-square test in case of different variables. The significance of the difference between the three groups was assessed by one-way analysis of variance (ANOVA) in case of normal distribution or by Kruskal-Wallis test (non-parametric variance analysis) in case of non-normal distribution of continuous variables. Differences between two groups were determined by Bonferroni post hoc test. $\mathrm{P}$ values less than 0.05 were accepted for statistical significance. All statistical analyzes were performed with SPSS statistical software (Version 22.0, SPSS Inc., Chicago, IL, USA).

\section{Results}

Of the 76 patients, 48 were males and 28 were females. Twentytwo men were in the low-risk, 16 were in the moderate-risk and 10 were in the high-risk groups for recurrence. Twenty-six men were in the low-risk, 14 were in the moderate-risk and eight were in the high-risk groups for progression. Twelve women were in the low-risk, 10 were in the moderate-risk and six were in the high-risk groups for recurrence. Fifteen women were in the low-risk, eight were in moderate-risk and five were in the high-risk groups for progression. The mean age of the high-risk group was higher than the low- and medium-risk groups (52.8 vs 56.6 vs 61.4 for the recurrence, 53.9 vs 58.8 vs 63.2 for progression, $p=0.026$ ). In addition, the mean tumor size and number of tumors were higher in the high-risk group for both the risk of recurrence and progression $(p=0.019$ and $p=0.032$, respectively). Demographic characteristics and comparison of tumor characteristics of patients according to the recurrence and progression groups is summarized in Table 1 .

Patients with both high risk of progression and recurrence had higher WBC values than patients with low- and moderate-risk ( 4.8 vs 5.8 vs 7.5 and 5.5 vs 6.8 vs $7.3, p=0.0027$ for progression and recurrence, respectively). According to post hoc test results, WBC values of moderate-risk group were higher than low-risk group ( $p=0.0041)$. The RBC, hemoglobin and hematocrit values of the high-risk group were lower than the other two groups $(p=0.0038, p=0.011$ and $p=0.0042$, respectively). The same values were lower in the moderate-risk group than the low-risk group ( $p=0.031, p=0.029$ and $p<0.001$, respectively). There were no significant differences in $\mathrm{MCV}, \mathrm{MCH}$ and MPV values between the three groups in both risk groups.

Neutrophil-lymphocyte ratio in both risk groups was significantly higher in the high-risk group than in the low- and moderaterisk groups $(p<0.001)$. Furthermore, according to the post hoc results, NLR values were significantly different in the highmoderate- and moderate-low-risk groups (high-moderate- and moderate-low- values for the risk of recurrence were 4.66 vs 3.67 and 3.67 vs $2.88, \mathrm{p}<0.001$, respectively, and moderatelow values were 4.72 vs 3.68 and 3.68 vs 2.92, $p<0.001$, respectively). A comparison of the blood parameters of patients in high-, moderate- and low-risk progression and recurrence groups is summarized in Table 2.

\section{Discussion}

Non-muscle-invasive bladder tumors, which constitute the majority of bladder tumors (75\%), constitute a heterogeneous tumor group with different recurrence, progression and diseaserelated mortality rates (15). The treatment methods planned according to the risk groups, physician and patient preferences vary significantly. Tumors in this group have a recurrence rate of up to $70-80 \%$ and a significant rate of progression (16). Therefore, identifying patients with similar risk of recurrence and progression in these patients is very important to predict the course of the disease and oncologic outcomes, and to decide the appropriate treatment method for each patient. For this purpose, the risk classification system developed by EORTC is widely used and patients can be grouped according to the risk of recurrence and progression by one and five years according to tumor characteristics (5). Based on the risk classification of the EORTC, the International Bladder Cancer Group and the EAU Panel have developed classification systems to divide patients into low, moderate, high, and very high-risk groups to guide treatments $(14,17)$. However, despite all efforts, the predictive level of these models with full accuracy is suboptimal to decide on optimal treatment (3). Due to the heterogeneous nature of the disease, factors are needed to predict the success of treatment and help to select the most appropriate treatment for each patient.

In recent years, the effect of inflammation on cancers has been investigated in general, and the mechanisms of carcinogenesis, progression, recurrence, metastasis and resistance have been tried to be elucidated (18). In this regard, NLR is one of the most important prognostic markers of inflammation. A recent meta-analysis of four studies with NMIBT and 14 studies with MIBT showed that preoperative NLR was associated with recurrence-free $(H R=1.58)$ and progression-free survival $(H R=1.33)(19)$. In the literature, there are few studies on the prognostic significance of NLR in MIBT and there is limited number of studies evaluating the relationship with NMIBT $(10,20,21)$.

The meta-analysis of six studies with a total of 2.298 patients demonstrated that NLR level in patients who underwent TURB because of NMIBT was a risk factor for increased disease recurrence and progression. In addition, NLR has been reported to be an independent predictor of disease recurrence and progression in NMIBT patients receiving BCG treatment (22). Identifying patients at risk for recurrence and progression in NMIBT patients contributes to the selection of the most appropriate candidates for treatment method such as intravesical BCG therapy or a more invasive radical cystectomy and optimization of the follow-up protocol of these patients. These results should be supported by well-designed, prospective randomized studies. There is no generally accepted NLR threshold-value in studies. Most researchers used the thresholdvalue determined by the highest specificity and sensitivity determined by the statistical method used, while others used threshold-values previously defined in the literature. In our study, NLR values differed significantly regarding prognostic groups, and unlike previous studies, a specific threshold-value was not used for NLR. In general, the threshold-value accepted or determined was over two.

$\mathrm{BT}$ is known to be an immunogenic malignancy and intravesical 
BCG is widely used for its treatment. BCG treatment has been shown to reduce the risk of recurrence and progression, particularly in the high-risk NMIBT group. The immunological system has an antagonistic effect in the pathogenesis of BT, while the acquired immune system has an anti-tumor effect, and the hereditary immune system has a pro-tumor effect (23). High NLR is believed to contribute to carcinogenesis by promoting tumor aggression by increasing the number of neutrophils by interacting with other groups of cells, producing cytokines and effector molecules. Neutrophils are capable of rapidly generating host responses by chemokines, pathogenic signals and lipid mediators. Major tumorigenic effects include cell invasion, cancer cell proliferation, lymphangiogenesis, and re-generation of the matrix outside the cell. On the other hand, anti-or pro-tumor effects of neutrophils, which are highly mobile cells, may also vary according to their microenvironment (24).

Mano et al. (25) evaluated the prognostic significance of NLR in 122 NMIBT patients who underwent TURB in their study and found that NLR over 2.41 was associated with disease progression and above 2.43 was associated with disease recurrence. In this study, similar to our study, the patients were classified according to the risk groups of EORTC and it was found that the number of patients with high NLR was higher in the high-risk group both in the progression and recurrence groups. The relationship between NLR and subgroups of T stage at the time of diagnosis was also evaluated and it was shown that NLR of patients with lamina propria invasive histopathology (T1) was higher than the non-invasive (Ta) group and that lymphocyte count was lower (26). In a retrospective study of the data of 1,551 patients who underwent TURB with a diagnosis of NMIBT in a single center from Korea, it was shown in multivariate analysis that high NLR is an independent predictor for both general and cancer-specific survival and may be an important predictor of oncologic outcomes, especially mortality (27).

In a study in 222 patients with MIBT and NMIBT, Celik et al. (28) concluded that NLR is a predictive biomarker and found significantly higher NLR in the MIBT group than in the NMIBT group. In addition, $\mathrm{RBC}, \mathrm{Hb}$ and Htc values were lower in the MIBT group. In our study, although all patients had NMIBT, these parameters were similarly significantly lower in the high-risk group. The fact that patients in the high-risk group complain more frequently of hematuria because they have more and larger tumors is the likely cause of this difference. These patients require more frequent hospitalization due to massive hematuria and are more frequently confronted with erythrocyte transfusion.

Recently, the effect of tumor-infiltrating immune cells on BT prognosis has been the subject of research. It has been demonstrated that tumor-infiltrating neutrophils and NLR are negative predictors and tumor-infiltrating lymphocytes are positive predictors (29). Ethnicity has also been shown to affect NLR. It was shown that NLR was associated with advanced tumor stage in 297 patients with NLR evaluation during TURB, but that European patients had higher NLR than African patients, and it was underlined that ethnicity is a factor to consider when interpreting NLR (30).

\section{Study Limitations}

There are some limitations of our study. The retrospective nature of our study and the relatively low number of patients in the groups are the main ones. The follow-up period of the patients was not long enough, but there was sufficient time for risk classification. In addition, because the NLR is a marker differentiating according to ethnicity, the results of this study with our own population should be carefully adapted to other ethnic groups. On the other hand, we believe that our study is valuable because it is one of the rare studies in the literature in which NLR classification of NMIBT patients according to EORTC risk groups is made.

\section{Conclusion}

The discovery of new predictive factors that accurately predict the prognosis of NMIBT, a highly heterogeneous group of patients, seems to be an important necessity in the field of urological oncology. Neutrophil-lymphocyte ratio is a promising, cost-effective and rapid biomarker in this regard, which is routinely applied in the pre-operative evaluation. In our study, we found that the groups with higher risk of recurrence and progression had higher NLR values in NMIBT patients. Neutrophil-lymphocyte ratio can be used in prognostic classification of these patients, in determining the treatment method and in estimating the state of muscle-invasion. In order to determine the potential role of NLR in the clinical decision stage, well-designed, large-scale, prospective studies with longterm follow-up are needed.

\section{Ethics}

Ethics Committee Approval: Because of the study was designed as a retrospective study, ethics committee approval was not obtained.

Informed Consent: Written informed consent was taken from all patients in order to be able to use their data in scientific studies without revealing their private information.

Peer-review: Externally peer-reviewed.

\section{Authorship Contributions}

Surgical and Medical Practices: F.K., A.Ş., Concept: F.K., A.Ş., Design: F.K., A.Ş., Data Collection or Processing: F.K., Analysis or Interpretation: F.K., A.Ş., Literature Search: F.K., Writing: F.K.

Conflict of Interest: No conflict of interest was declared by the authors.

Financial Disclosure: The authors declared that this study received no financial support.

\section{References}

1. Torre LA, Bray F, Siegel RL, et al. Global cancer statistics, 2012. CA Cancer J Clin 2015;65:87-108.

2. Compérat $E$, Larré $S$, Roupret $M$, et al. Clinicopathological characteristics of urothelial bladder cancer in patients less than 40 years old. Virchows Arch 2015;466:589-594.

3. Sylvester RJ, van der Meijden AP, Oosterlinck W, et al. Predicting recurrence and progression in individual patients with stage Ta T1 bladder cancer using EORTC risk tables: a combined analysis of 2596 patients from seven EORTC trials. Eur Urol 2006;49:466-465. 
4. Fernandez-Gomez J, Madero R, Solsona E, et al. Predicting nonmuscle invasive bladder cancer recurrence and progression in patients treated with bacillus Calmette-Guerin: the CUETO scoring model. J Urol 2009; 182:2195-2203.

5. Cambier S, Sylvester RJ, Collette L, et al. EORTC Nomograms and Risk Groups for Predicting Recurrence, Progression, and Disease-specific and Overall Survival in Non-Muscle-invasive Stage Ta-T1 Urothelial Bladder Cancer Patients Treated with 1-3 Years of Maintenance Bacillus Calmette-Guérin. Eur Urol 2016;69:60-69.

6. Sarraf KM, Belcher E, Raevsky E, et al. Neutrophil/lymphocyte ratio and its association with survival after complete resection in non-small cell lung cancer. J Thorac Cardiovasc Surg 2009;137:425-428.

7. Walsh S, Cook EJ, Goulder F, et al. Neutrophil lymphocyte ratio as a prognostic factor in colorectal cancer. J Surg Oncol 2005;91:181-184.

8. Stotz M, Gerger A, Eisner F, et al. Increased neutrophil-lymphocyte ratio is a poor prognostic factor in patients with primary operable and inoperable pancreatic cancer. Br J Cancer 2013;109:416-421.

9. Shimada H, Takiguchi N, Kainuma $\mathrm{O}$, et al. High preoperative neutrophil-lymphocyte ratio predicts poor survival in patients with gastric cancer. Gastric Cancer 2010;13:170-176.

10. Gondo T, Nakashima J, Ohno Y, et al. Prognostic value of neutrophilto-lymphocyte ratio and establishment of novel preoperative risk stratification model in bladder cancer patients treated with radical cystectomy. Urology 2012;79:1085-1091.

11. Demirtaş A, Sabur V, Akınsal EC, et al. Can neutrophil-lymphocyte ratio and lymph node density be used as prognostic factors in patients undergoing radical cystectomy? ScientificWorldJournal 2013;2013:703579

12. Potretzke A, Hillman L, Wong $K$, et al. NLR is predictive of upstaging at the time of radical cystectomy for patients with urothelial carcinoma of the bladder. Urol Oncol 2014:631-636.

13. Babjuk M, Böhle A, Burger M, et al. EAU Guidelines on Non-Muscleinvasive Urothelial Carcinoma of the Bladder: Update 2016. Eur Urol 2017;71:447-461.

14. Alfred Witjes J, Lebret T, Compérat EM, et al. Updated 2016 EAU Guidelines on Muscle-invasive and Metastatic Bladder Cancer. Eur Urol 2017;71:462-475.

15. Sylvester RJ, van der Meijden AP, Oosterlinck W, et al. Predicting recurrence and progression in individual patients with stage Ta T1 bladder cancer using EORTC risk tables: a combined analysis of 2596 patients from seven EORTC trials. Eur Urol 2006;49:466-467.

16. Witjes JA, Compérat E, Cowan NC, et al. EAU guidelines on muscleinvasive and metastatic bladder cancer: summary of the 2013 guidelines. Eur Urol 2014;65:778-792.

17. Brausi M, Witjes JA, Lamm D, et al. A review of current guidelines and best practice recommendations for the management of nonmuscle invasive bladder cancer by the International Bladder Cancer Group. J Urol 2011;186:2158-2167.

18. Diakos Cl, Charles KA, McMillan DC, et al. Cancer-related inflammation and treatment effectiveness. Lancet Oncol 2014;15:e493-503.

19. Tang X, Du P, Yang Y. The clinical use of neutrophil-to-lymphocyte ratio in bladder cancer patients: a systematic review and metaanalysis. Int J Clin Oncol 2017;22:817-825.

20. Can C, Baseskioglu B, Yllmaz M, et al. Pretreatment Parameters Obtained from Peripheral Blood Sample Predicts Invasiveness of Bladder Carcinoma. Urol Int 2012;89:468-472.

21. Ceylan C, Doluoglu OG, Keleş I, et al. Importance of the neutrophilto-lymphocyte ratio in muscle-invasive and non-muscle invasive bladder tumors. Urologia Journal 2014;81:120-124.

22. Vartolomei MD, Porav-Hodade $D$, Ferro $M$, et al. Prognostic role of pretreatment neutrophil-to-lymphocyte ratio (NLR) in patients with non-muscle-invasive bladder cancer (NMIBC): A systematic review and meta-analysis. Urol Oncol; 2018;36:389-399.

23. Thompson DB, Siref LE, Feloney MP, et al. Immunological basis in the pathogenesis and treatment of bladder cancer. Expert Rev Clin Immunol 2015;11:265-279.

24. Fridlender ZG, Sun J, Kim S, et al. Polarization of tumor-associated neutrophil phenotype by TGF-beta: "N1" versus "N2" TAN. Cancer Cell 2009;16:183-194.

25. Mano R, Baniel J, Shoshany O, et al. Neutrophil-to-lymphocyte ratio predicts progression and recurrence of non-muscle-invasive bladder cancer. Urol Oncol 2015:67.e1-e7.

26. Cimen HI, Halis F, Saglam HS, et al. Can neutrophil to lymphocyte ratio predict lamina propria invasion in patients with non muscle invasive bladder cancer? Int Braz J Urol 2017;43:67-72.

27. Kang $M$, Jeong CW, Kwak C, et al. Preoperative neutrophil-lymphocyte ratio can significantly predict mortality outcomes in patients with non-muscle invasive bladder cancer undergoing transurethral resection of bladder tumor. Oncotarget 2017;8:12891-12901.

28. Celik O, Akand M, Keskin M, et al. Preoperative neutrophil-tolymphocyte ratio (NLR) may be predictive of pathologic stage in patients with bladder cancer larger than $3 \mathrm{~cm}$. Eur Rev Med Pharmacol Sci 2016;20:652-656.

29. Liu K, Zhao K, Wang L, et al. The prognostic values of tumor-infiltrating neutrophils, lymphocytes and neutrophil/lymphocyte rates in bladder urothelial cancer. Pathol Res Pract 2018;214:1074-1080.

30. Tazeh NN, Canter DJ, Damodaran S, et al. Neutrophil to Lymphocyte Ratio (NLR) at the Time of Transurethral Resection of Bladder Tumor: A Large Retrospective Study and Analysis of Racial Differences. Bladder Cancer 2017;3:89-94. 